RU9562

\title{
Critical point multiplicities and multiplicity fluctuations in heavy ion collisions
}

\author{
K. C. Chase and A. Z. Mekjian \\ Department of Physics, Rutgers University \\ Piscataway, New Jersey 08855 USA
}

(October 26, 2018)

\begin{abstract}
An exactly solvable model of nuclear fragmentation is shown to lead to a simple connection between the critical point multiplicity $\langle m\rangle_{c}$ and the critical point exponent $\tau$ recently reported on in the EOS collaboration. The importance of multiplicity fluctuations on critical point behavior is also discussed. 05.70.Jk, 25.70.Pq

KEYWORDS: Nuclear Fragmentation, Critical Phenomena, Multiplicity Fluctuations
\end{abstract}

Typeset using REVTEX 
In a recent paper [1] the EOS collaboration reported some very interesting results on the critical point behavior of nuclear matter. The analysis of the data is based on percolation model predictions due to Campi [2]. In this analysis, three parameters appear, one being the expected critical multiplicity $\langle m\rangle_{c}$ and the other two critical exponents $\gamma$ and $\beta$. The $\gamma$ and $\beta$ exponents are then used to obtain the critical exponent $\tau$. The purpose of this paper is to show there is a simple connection between the critical exponent $\tau$ and the critical multiplicity $\langle m\rangle_{c}$. The development of this relation is based on an exactly solvable statistical model of hadronic matter which shares some features with percolation models. Namely, in the infinite mass number $A$ limit, an infinite cluster can appear below a critical point in a variable $x$ called the tuning parameter, just as in percolation models an infinite cluster may exist only at and above a critical site or bond probability $p_{c}$. Thermodynamic and statistical arguments relate the tuning parameter $x$ to the volume, temperature, binding energy coefficient $a_{V}$, and level spacing parameter $\varepsilon_{0}$, namely

$$
x=\frac{V}{v_{0}(T)} \exp \left\{-\frac{a_{V}}{k_{B} T}-\frac{k_{B} T}{\varepsilon_{0}} \frac{T_{0}}{T+T_{0}}\right\} .
$$

Further details can be found in Ref. [3].

A parallel of the exactly solvable model of hadronic matter with Bose condensation and Feynman's approach to the $\lambda$ transition in liquid helium was also noted in previous studies [3]. In this parallel, the cluster size in fragmentation is equivalent to the cycle length in the cycle class decomposition of the symmetric group for the Bose problem. The number of clusters of size $k$ is the same as the number of cycles of length $k$. The complete fragmentation into nucleons corresponds to all unit cycles, which is the identity permutation. Bose condensation corresponds to the appearance of longer and longer cycles in the cycle class decomposition of the symmetric group.

In the statistical model of Ref. [0] ] each fragmentation outcome happens with a probability proportional to the Gibbs weight

$$
P(\vec{n})=\frac{1}{Z_{A}(\vec{x})} \prod_{k \geq 1} \frac{x_{k}^{n_{k}}}{n_{k} !}
$$


where $Z_{A}(\vec{x})$ is the canonical partition function. Here $x_{k}=x / k^{\tau}$ with $x$ as given above and $\tau$ the critical exponent originally introduced in nuclear fragmentation by Finn, et al. 四. Cluster distributions can be obtained from the partition functions $Z_{A}(\vec{x})$ using

$$
\left\langle n_{k}\right\rangle=x_{k} \frac{Z_{A-k}(\vec{x})}{Z_{A}(\vec{x})}
$$

In turn the $Z_{A}(\vec{x})$ can be obtained by recursive techniques, and in particular

$$
Z_{A}(\vec{x})=\frac{1}{A} \sum_{k \geq 1} k x_{k} Z_{A-k}(\vec{x})
$$

with $Z_{0}(\vec{x})=1$. If $A$ is very large we can work in the grand canonical limit, where $\left\langle n_{k}\right\rangle=$ $x z^{k} / k^{\tau},\langle m\rangle=x g_{\tau}(z)$ and $A=x g_{\tau-1}(z)$. Here $z \leq 1$ is the fugacity and $g_{\tau}(z)=\sum_{k} z^{k} / k^{\tau}$. At $z=1, g_{\tau-1}(z)$ is finite only if $\tau>2$, which indicates a critical point at $A=x_{c} g_{\tau-1}(1)$. At this point the expected multiplicity $\langle m\rangle_{c}$ is given by

$$
\frac{\langle m\rangle_{c}}{A}=\frac{\zeta(\tau)}{\zeta(\tau-1)}
$$

where $\zeta(x)$ is the Riemann zeta function.

The EOS collaboration recently reported independent determinations of $\langle m\rangle_{c}$ and $\tau$. Specifically, they found $\langle m\rangle_{c}=26 \pm 1$ and $\tau=2.14 \pm 0.06$ for the fragmentation of gold nuclei by using a percolation model analysis. For our model, $\langle m\rangle_{c}=26 \pm 1$ implies a critical exponent $\tau=2.262 \pm 0.013$ which is consistent with EOS result, but based on a different model.

The critical behavior of this model is most clearly seen from the behavior of the multiplicity fluctuations $\left\langle m^{2}\right\rangle-\langle m\rangle^{2} \equiv\langle m\rangle_{2}$. In the grand canonical limit, these fluctuations are given by

$$
\langle m\rangle_{2}=\left\{\begin{array}{ll}
\langle m\rangle & \langle m\rangle \leq\langle m\rangle_{c} \\
\langle m\rangle-x \frac{g_{\tau-1}(z)^{2}}{g_{\tau-2}(z)} & \langle m\rangle>\langle m\rangle_{c}
\end{array},\right.
$$

which is continuous function with a maximum at the transition point. However the slope of $\langle m\rangle_{2}$ is discontinuous at the transition point, i.e. the peak at the critical point is a cusp. Specifically, 


$$
\left.x \frac{\partial}{\partial x}\langle m\rangle_{2}\right|_{x \rightarrow x_{c}^{+}}-\left.x \frac{\partial}{\partial x}\langle m\rangle_{2}\right|_{x \rightarrow x_{c}^{-}}=-2 A x_{c}
$$

Such a discontinuity is indicative of a phase transition. For finite $A$, the exactly solvable model developed in Ref. [3] leads to a rounded peak, which is shown in Fig. 1 1 which is a plot of $\langle m\rangle_{2} / A$ vs. $\langle m\rangle / A$ for $\tau=2.5$.

This behavior of the multiplicity fluctuation arises from the sudden appearance of an infinite cluster below the critical point, a situation that parallels a similar result in percolation models. In percolation theory an infinite cluster exists only when the site or bond probability exceeds a critical value as noted above. For the nuclear fragmentation model, the critical condition and the sudden presence of an infinite cluster also parallels the discussion of Bose condensation. Specifically, the Bose condensation phenomenon is equivalent to a condensation of nucleons into the largest cluster and the argument for the presence of the infinite cluster is the same as that used to discuss the appearance of the Bose condensed state. Namely, since $A / x=\sum_{k} z^{k} k^{1-\tau}$ where $x \propto V$, then for $z<1$, the sum $\sum_{k} z^{k} k^{1-\tau}$ is finite. At $z=1$, the sum is finite only for $\tau>2$ and for $z>1$ the sum will diverge; hence the sum must be truncated. This truncation implies the expectation of certain large clusters must be zero. For $\tau \leq 2$ an infinite cluster does not exist which is equivalent to the condition that Bose condensation does not exist in two or fewer dimensions.

In summary, an exactly solvable statistical model of heated hadronic matter leads to a relationship between the critical multiplicity $\langle m\rangle_{c}$ and critical exponent $\tau$ which characterizes the power law fall off of cluster yields with mass number. The critical multiplicity can be obtained from the peak in the multiplicity fluctuations, which in the infinite $A$ limit has a cusp behavior due to the sudden appearance of an infinite cluster in the theory.

This work supported in part by the National Science Foundation Grant No. NSFPHY 92-12016. 


\section{REFERENCES}

[1] M. L. Gilkes, et. al., Phys. Rev. Lett. 73, 1590 (1994); J. B. Elliott, et. al. Phys. Rev. C 49, 3185 (1994).

[2] X. Campi, J. Phys. A 19, L917 (1986).

[3] K. C. Chase and A. Z. Mekjian, Phys. Rev. C 49, 2164 (1994). A. Z. Mekjian, Phys. Rev. Lett. 64, 2125 (1990); Phys. Rev. C 41, 2103 (1990); S. J. Lee and A. Z. Mekjian, Phys. Rev. C 45, 365 (1992); Phys. Rev. C 45, 1284 (1992).

[4] J. E. Finn et al., Phys. Rev. Lett. 49, 1321 (1982). 


\section{FIGURES}

FIG. 1. $\langle m\rangle_{2} / A$ vs. $\langle m\rangle / A$ for $x_{k}=x / k^{5 / 2}$. The function reaches a maximum at the critical point in the infinite $A$ limit. 
This figure "fig1-1.png" is available in "png" format from: http://arxiv.org/ps/nucl-th/9510005v1 